\title{
Microanalysis of transition metal substituted ZnO
}

\author{
S. Kolesnik, * B. Dabrowski, ${ }^{*}$ and R. E. Cook, ** \\ *Department of Physics, Northern Illinois University, DeKalb, IL 60115, U.S.A. \\ **Electron Microscopy Center, Argonne National Laboratory, Argonne, IL 60439, U.S.A.
}

Theoretical predictions of carrier-induced room temperature ferromagnetism in p-type Mnsubstituted $\mathrm{ZnO}$ (among other materials) by Dietl et al. [1] pointed to a possible novel application of this material in the field of spintronics. Since then, the presence or absence of room temperature ferromagnetism in transition metal substituted $\mathrm{ZnO}$ were alternatively reported by many groups. Sharma et al. [2] have claimed that $\mathrm{Mn}$ substituted $\mathrm{ZnO}$ can be obtained homogeneous and uniform from the low-temperature $500-700^{\circ} \mathrm{C}$ ceramic processing.

We have verified those findings by synthesis and structural, compositional and magnetic studies of low-temperature processed $(\mathrm{Zn}, \mathrm{Mn}) \mathrm{O}$ [3]. The cation ratio in this material was determined by energy dispersive x-ray spectroscopy (EDXS) analysis in a Hitachi S-4700-II scanning electron microscope. Typically, 15-20 spot spectra were collected across the surface of sintered pellets. Our results demonstrate that this compound can be formed in air at temperatures higher than $900^{\circ} \mathrm{C}$ using a ceramic route. Above this temperature, the crystallographic axes start to expand in agreement with substitution of larger $\mathrm{Mn}$ ions for $\mathrm{Zn}$ ions as well as the increase of the effective Mn content, determined from EDXS analysis can be observed (Fig. 1). Low-temperature annealing leaves an incompletely reacted mixture of $\mathrm{ZnO}$ and manganese oxides. Loose grains of a manganese oxide can be observed in an SEM micrograph, presented in Fig. 2. No bulk ferromagnetism can be observed for any of the studied samples, which show paramagnetic properties analogous to other diluted magnetic semiconductors [4].

The effective transition metal ion contents determined for transition metal (TM) substituted $\mathrm{ZnO}$ with $\mathrm{TM}=\mathrm{Mn}, \mathrm{Co}$, and $\mathrm{Fe}$ are presented in Fig. 3. For $\mathrm{TM}=\mathrm{Co}$, we observe a good agreement between the effective $\left(\mathrm{x}_{\mathrm{eff}}\right)$ and nominal $(\mathrm{x})$ TM contents, within the experimental error. For TM $=\mathrm{Mn}, \mathrm{x}_{\mathrm{eff}} \sim \mathrm{x}$ for $\mathrm{x}<=0.05$ and decreases for larger nominal $\mathrm{x}$. For $\mathrm{TM}=\mathrm{Fe}, \mathrm{x}_{\mathrm{eff}} \sim \mathrm{x}$ for $\mathrm{x}=$ 0.05 .

Our results show the significance of compositional studies in the determination of the homogeneity of novel oxide materials. By combining this method with the x-ray diffraction analysis and magnetic measurements, we are able to determine the magnetic properties of singlephase $\mathrm{ZnO}$-based magnetic semiconductors. Further studies are required to introduce p-type doping in this material, which appears to be crucial for the existence of room-temperature ferromagnetic order. The EDXS analysis is a powerful tool, which will assist in the investigation of prospective novel materials including transition metal substituted $\mathrm{ZnO}$.

Work was supported by the NSF grant no. DMR-0302617.

[1] T. Dietl, H. Ohno, F. Matsukura, J. Cibert, and D. Ferrand, Science 287 (2000) 1019.

[2] P. Sharma, A. Gupta, K. V. Rao, F. J. Owens, R. Sharma, R. Ahuja, J. M. Osorio Guillen, B. Johansson, and G. A. Gehring, Nature Mater. 2 (2003) 673.

[3] S. Kolesnik, and B. Dabrowski, J. Appl. Phys. 96 (2004) 5379.

[4] S. Kolesnik, B. Dabrowski, and J. Mais, J. Appl. Phys. 95 (2004) 2582.

[5] Results shown here are derived from work at Argonne National Laboratory, which is operated by the U. of Chicago for the U. S. Dept. of Energy under contract W-31-109-Eng-38. 


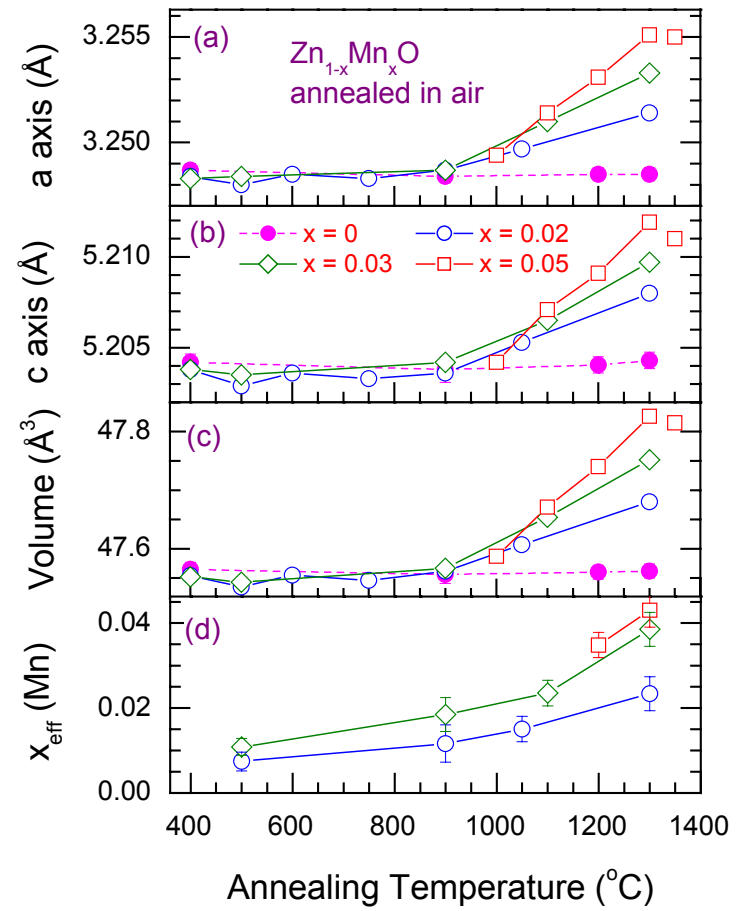

Fig. 1. (a-c) Lattice parameters for $\mathrm{Zn}_{1-\mathrm{x}} \mathrm{Mn}_{\mathrm{x}} \mathrm{O}$ annealed in air at various temperatures, $(\mathrm{d})$ The effective Mn content determined from the EDXS. Open symbols: substituted samples, filled circles: pure $\mathrm{ZnO}$.

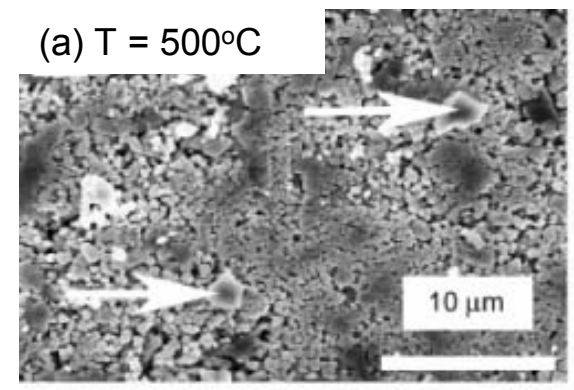

(c) $\mathrm{T}=1100^{\circ} \mathrm{C}$

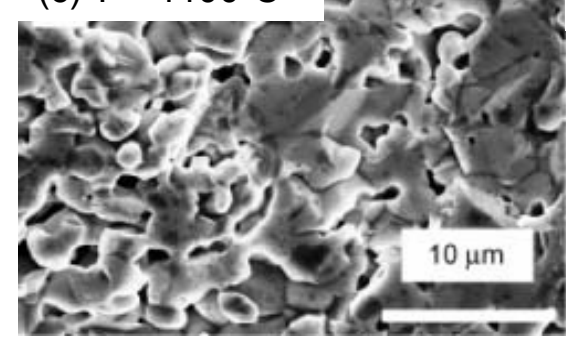

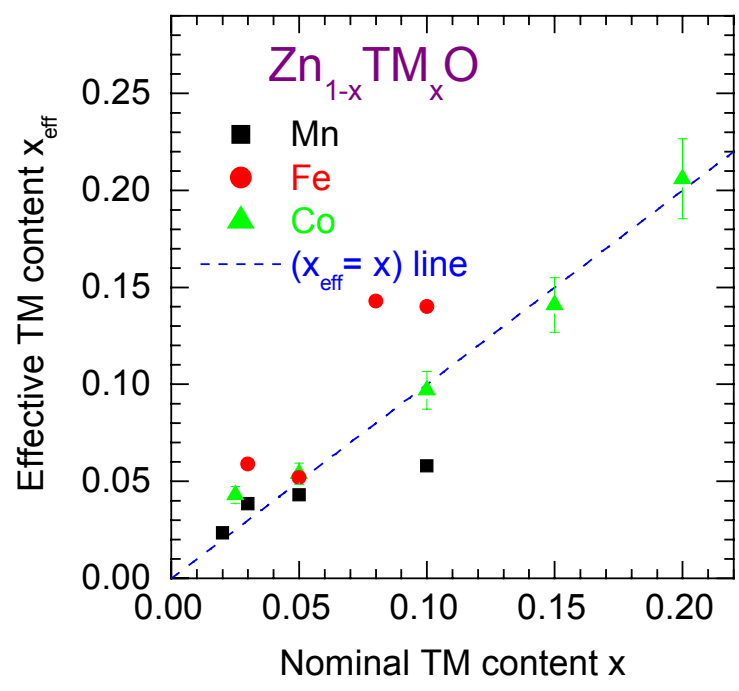

Fig. 3. The effective TM content in $\mathrm{Zn}_{1-\mathrm{x}} \mathrm{TM}_{\mathrm{x}} \mathrm{O}$ determined from the EDXS analysis.

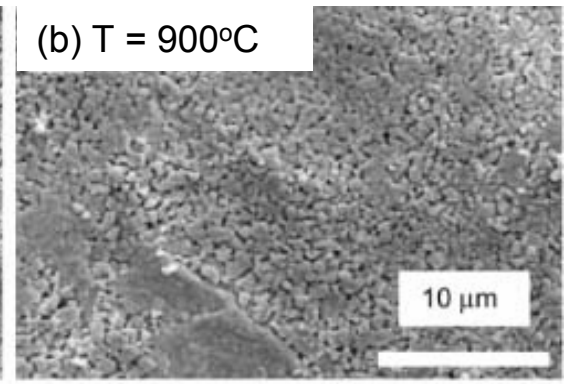

(d) $\mathrm{T}=1300^{\circ} \mathrm{C}$

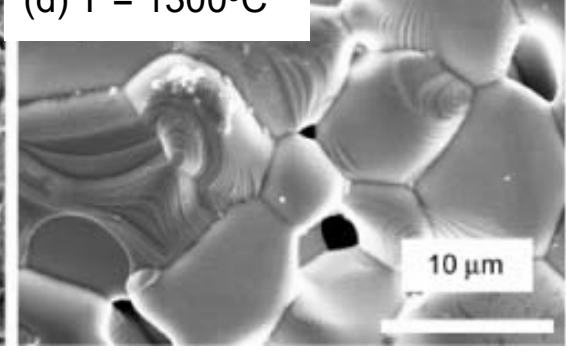

Fig. 2. Scanning electron micrographs of $\mathrm{Zn}_{0.97} \mathrm{Mn}_{0.03} \mathrm{O}$ pellets after annealing in air at (a) $500^{\circ} \mathrm{C}$, (b) $900^{\circ} \mathrm{C}$, (c) $1100^{\circ} \mathrm{C}$, and (d) $1300^{\circ} \mathrm{C}$. Arrows in (a) indicate grains of pure manganese oxide. 